\title{
New high-density deformable mirrors for high-contrast imaging
}

\author{
P. Riaud ${ }^{1,2}$
}

\author{
160 rue des bergers, 75015 Paris, France \\ e-mail: riaud.pierre@gmail.com \\ ${ }^{2}$ Université de Liège, 17 Allée du 6 Août, 4000 Sart Tilman, Belgium
}

Received 29 May 2012 / Accepted 25 July 2012

\begin{abstract}
This paper presents a new architecture for deformable mirrors, suitable for adaptive optics (AO) observations. The performance of these systems directly depends on the density of actuators, which correct for wavefront errors. Therefore, deformable mirrors of large sizes are difficult to build because of the required number of actuators. Moreover, these actuators use either piezoelectric, magnetic, or electrostatic systems, which all suffer from nonlinear behavior (hysteresis). To solve these problems, we propose to use addressable vertical-cavity surface-emitting laser (VCSELs) systems, which move, through radiation pressure, a CVD-diamond membrane coated with an aluminum/gold thin film. The advantage of such a system is the high actuator density (16384 on $\left.41 \mathrm{~mm}^{2}\right)$ without hysteresis problems. This technology is therefore well-suited for the next generation of AO systems.
\end{abstract}

Key words. instrumentation: high angular resolution - instrumentation: adaptive optics - methods: numerical

\section{Introduction}

Deformable mirrors (DM), coupled with wavefront-sensing systems, are used in adaptive optics to correct in real time for the atmospheric turbulence. They are also used in high-contrast imaging optical workbenches such as HCIT (Trauger 2009) to correct for some residual speckles after some coronagraphics systems. Indeed, phase and intensity errors introduced in the pupil plane create residual speckles in the final imaging plane after the coronagraphic effect. All coronagraphs suppress the coherent part of the light and leave some residual non-coherent speckles.

Extreme AO (ExAO) can be used to correct these phase residuals at the $0.1 \mathrm{~nm}$ level. In these cases, using high-density deformable mirrors with a small footprint (active area) is essential to correct for the high number of Zernike polynomials, without hysteresis. Nowadays, the performance of state-of-theart high-density DMs are still limited by their relatively significant footprint $\left(>9 \mathrm{~cm}^{2}\right)$. Despite the technological advances, new techniques that use piezoelectric, magnetic, or electrostatic systems generate undesirable hysteresis responses.

In this paper, we present a new concept that solves most of these drawbacks (density, footprint, hysteresis). The solution we propose makes use of high-density vertical-cavity surfaceemitting laser (VCSELs) that move a thin CVD-diamond membrane (e.g., synthetic diamond, produced using chemical vapor deposition technique) with an aluminum coating through radiation pressure. Thanks to present progress in the microsystem manufacturing, a $128 \times 128$ fully matrix-addressable VCSELs array is under study today. This configuration allows high actuator density (16384), small footprint $\left(0.41 \mathrm{~cm}^{2}\right.$ with $50 \mu \mathrm{m}$ array pitch) and no hysteresis due to the free-mechanical movement induced by each laser radiation pressure.

The DM concept is explained in detail in Sect. 2, while the radiation pressure theory on a clamped membrane is presented in Sect. 3. Because the device uses a VCSELs array (Kasten et al. 2007; Seurin et al. 2008; Bardinal et al. 2011) to exercise pressure on the thick membrane via laser beams, it is essential to take into account any possible diffraction effects; these calculations are given in Sect. 4. The optical propagation of light allows one to calculate (a) the "waffle" effect and (b) the inter-actuator crossing effect, because the laser beam size is larger than the actuator pitch (see the Gaussian convolution in Appendix E). The first stage of the numerical simulation of the membrane deflection can be performed with a simple linear model, which is presented in Sect. 5. However, due to the very thick (1-2 $\mu \mathrm{m})$ multilayer membrane, the deformation level can be higher than the thickness and a nonlinear model must also be investigated to perform better numerical simulations of the deformation constraint (i.e. laser power vs. dynamic). These calculations are presented in Sect. 5.5. The temporal response of the device is also investigated in Sect. 7 by directly calculating the resonance frequencies. Finally, an estimation of the thermal deformation of the membrane, due to the high laser power, is proposed in Sect. 8.

\section{Principle}

This paper introduces the concept of using radiation pressure to distort a thin CVD-diamond membrane. The pressure is produced by a fully addressable VCSELs array, which consists of spatially monomode (TEM 00) laser diodes. This new optical MEMS architecture creates a high-density actuator on a very small footprint. The impressive performance of VCSELs, such as (a) high-power laser-diode emission (several $\mathrm{mW}$ per diode), (b) circular monomode beam, and (c) efficient high-frequency modulation, is providing the motivation for using light for a possible non-mechanical movement of the thin membrane with radiation pressure (Mansuripur 2004; Labeyrie et al. 2010).

\subsection{VCSELs array manufacturing}

Up to $128 \times 128$ VCSELs array are under study in the near-infrared $(808-1064 \mathrm{~nm})$ and this high number of 
laser-diodes (16384) constitutes a possibility to obtain the largest high-density deformable mirror ever made. We propose to use laser-diodes working around $808-1064 \mathrm{~nm}$ because this technology allows a high-power emission (up to $1 \mathrm{KW}$ for an entire array, see for example the commercially available Lasertel product V1 QCW for high-power VCSEL at $808 \mathrm{~nm}$ ). The actuator pitch is a free parameter, but with the present nanotechnology the number of laser-diodes varies inversely with the pitch dimension, and a value between $25-100 \mu \mathrm{m}$ should be feasible. The main problem in manufacturing a fully-addressable VCSELs array is the number of metal connections or wires that is required to operate the array. Matrix-addressable arrays greatly reduce the number of required metal connections. As a comparison, for a $128 \times 128$ array, 16384 metal wires are required for the individually addressed array, but only 256 metal wires are required for the matrix-addressable array (Conk 2002). If we chose the distance between each laser diode to be $50 \mu \mathrm{m}$, the effective size of the deformable mirror will only be $6.5 \mathrm{~mm} \times 6.5 \mathrm{~mm}$. This footprint is perfectly adapted to classical optical workbenches used for high-contrast imaging. Indeed, in this type of workbench, the size of the pupil must remain small in diameter (with 0.25 to $3 \mathrm{~cm}$ ) to ensure a relatively small size of the overall optical system ( $2 \mathrm{~m}$ to $3 \mathrm{~m})$.

Each laser-diode can be driven in intensity with highfrequency modulation (several $\mathrm{KHz}$ ), to allow a proper distortion of a small part of the thin membrane. Before that, the Gaussian monomode beam provided by each microlaser must be collimated with an array of square fused silica microlenses. Let $\Lambda_{x}, \Lambda_{y}$ be the distance between each laser diode in the $x$ and $y$ direction, respectively, and $N d=n_{x} \times n_{y}$ the total number of laser-diodes. These parameters are important to define the actuator pitch and the full effective size of the deformable mirror. The focal length $F_{\mathrm{L}}$ needed for a good collimation is given directly by the beam divergence $\left(1 / e^{2}\right)$ :

$$
F_{\mathrm{L}}=\frac{\min \left(\Lambda_{x}, \Lambda_{y}\right)}{2 \tan \left(\left(1 / e^{2}\right) / 2\right)}
$$

We obtain 15 to 20 degrees of the total beam divergence for the commercial VCSELs array, the focal length of the microlens array needs to be $F_{\mathrm{L}}>142 \mu \mathrm{m}$ for a $50 \mu \mathrm{m}$ array pitch. This value is the minimum focal length to achieve a complete illumination of the microlens by the full width at half maximum of the Gaussian beam. To know the full laser field given by the entire array, we must simulate the propagation until the membrane is reached with Fresnel diffraction. The gap between microlens and a limited precision alignment must be taken into account to properly evaluate the diffraction field on the membrane, which produces some correlation problems between actuators. In classical mirror theory, this correlation is generally known as "waffle" effect.

Figure 1 presents the optical scheme that uses polarized light (provided directly by the VCSELs array or by a linear polarizing system) to separate the input laser beam $\left(E_{\text {in }}, H_{\text {in }}\right)$ from the output $\left(E_{\text {out }}, H_{\text {out }}\right)$ after reflection on the thin coated membrane. The proposed optical device can either be a Faraday rotator or a polarizing cube, necessary to achieve a perfect separation of the output beam and dramatically decrease a possible interference process between the two laser fields.

\subsection{Membrane manufacturing}

Finally, we decided to take a $1 \mu \mathrm{m}$ thick CVD-diamond membrane (see Espinosa et al. 2003; Koizumi et al. 2008) with

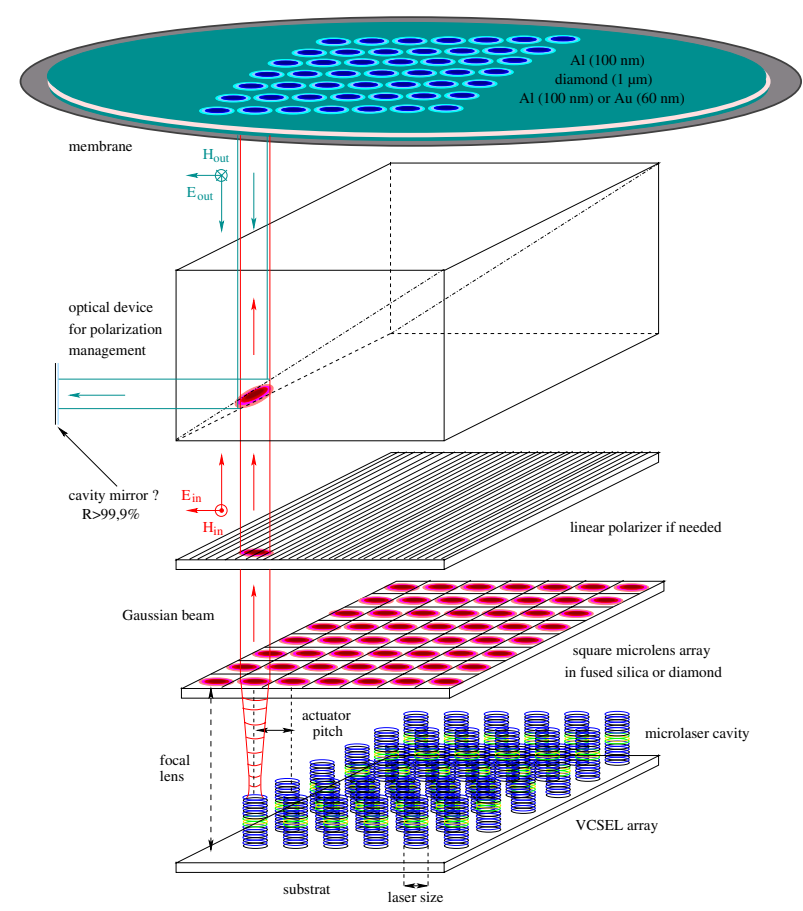

Fig. 1. Key functional elements of a high-density actuator deformable mirror, driven by radiation pressure from a VCSELs array. Bottom to top: fully addressable VCSELs array of laser diodes in the near-infrared (808-1064 nm). The emitting region of the laser diode is around $9 \mu \mathrm{m}$ (the laser size), and the actuator pitch is given by the laser diode spacing. Each Gaussian beam (provided by laser diodes) is collimated by a square microlens array in fused silica with a proper anti-reflection coating (transmission $>99.5 \%$ ). The microlens array must be very well aligned $(<0.5 \mu \mathrm{m})$ with the VCSEL array. If the VCSELs array is not in polarized emission, a linear polarizer is needed. The light passes through an optical polarizing device and arrives on a thin CVD-diamond membrane $(\approx 1 \mu \mathrm{m}$ of thickness) with aluminum coating $(100 \mathrm{~nm}$ of thickness) on each face. The linear polarizer and the optical polarizing device management can have the same component as a polarizing cube with high polarization purity $\left(>10^{4}\right)$. The radiation pressure produced by each laser diode locally distorts the membrane with a Gaussian shape, which definies the actuator response of the system.

a $100 \mathrm{~nm}$ aluminum and $60 \mathrm{~nm}$ gold coating. The thickness of the membrane must be (a) as low as possible to allow a sufficient displacement (i.e. dynamic range) due to the radiation pressure and (b) thin enough not to break during movement. The CVD-diamond material constitutes the best possible choice, due to its high Young's modulus (greater than $1000 \mathrm{GPa}$ ). The mechanical resonance frequency must be high compared to the maximum frequency $(10 \mathrm{KHz})$ used in the deformable mirror (200 KHz for a $50 \mu \mathrm{m}$ length).

To manufacture a diamond membrane of $1-10 \mu \mathrm{m}$ thickness range, we must use nanocrystalline or ultra-nanocrystalline CVD diamond (Hess 2012). Membranes as thin as $100 \mathrm{~nm}$ already exist (Espinosa et al. 2003) and clearly demonstrate the feasibility of the DM concept. The choice of reflection coating for the laser beams can be a simple aluminum, because it directly provides a low-density membrane $\left(3.51 \mathrm{~g} / \mathrm{cm}^{3}\right.$ for CVDdiamond material and $2.7 \mathrm{~g} / \mathrm{cm}^{3}$ for aluminum coating), but gold $\left(19.3 \mathrm{~g} / \mathrm{cm}^{3}\right)$ provides a better reflectivity at $980 \mathrm{~nm}$ than aluminum also with low-roughness and low-depolarization issue. The gold coating must be greater than $60 \mathrm{~nm}$ of thickness. Concerning the reflecting surface used for the phase corrections, the metallic coating can also be aluminum because it provides low roughness and small mass at the other face. 
In the following section we present the fundamental equations that describe the radiation pressure (the Lorentz law), using as a starting point Maxwell's electromagnetic field equations.

\section{Radiation pressure simulation}

\subsection{The Lorentz law}

Following Mansuripur (2004), we calculated the electromagnetic radiation force and its associated acceleration for a thin suspended membrane, produced by a direct application of the Lorentz law of classical electrodynamics. We used Maxwell's equations to determine the distributions of the two fields, $E$ and $H$, present in the electromagnetic radiation both inside and outside the considered medium. The Lorentz law describes the electromagnetic force $F$ on a particle of charge $q$ and velocity $\vec{V}$ :

$\vec{F}=q(\vec{E}+\vec{V} \times \vec{B})$

The magnetic field $\vec{B}$ is related to the H-field via the permeability of free space $\mu_{0}$,

$\vec{B}=\mu_{0} \vec{H} \quad \nabla \vec{B}=0$

All three $\vec{E}, \vec{B}, \vec{H}$ fields are classical time harmonic fields, written for the electric field as $\vec{E}(x, y, z, t)=\vec{E}(x, y, z) \exp (-\mathrm{i} \omega t)$ with $\omega$ the angular frequency.

The next step is to calculate the interaction of laser light in the considered medium. For this purpose we introduce two new vectors that take into account some of the properties of the interacting medium:

$\vec{D}=\epsilon_{0} \vec{E}+\vec{P} \quad \nabla \vec{D}=0$

where $\vec{D}$ is the electric displacement vector, $\vec{P}$ is the local polarization density within the considered medium, and $\epsilon_{0}$ is the permittivity of free space. The divergence of $\vec{D}$ is generally equal to zero and occurs when there are no free charges in the system.

In a linear medium with a relative permittivity $\epsilon$, the two vectors $\vec{D}, \vec{P}$ become

$\vec{D}=\epsilon_{0} \epsilon \vec{E} \quad \vec{P}=\epsilon_{0}(\epsilon-1) \vec{E}$

The complex refractive index of the medium is given by

$n+\mathrm{i} \kappa=\sqrt{\epsilon}$

When the light interacts with the medium, the perpendicular $H$-field at the interface between adjacent media must remain continuous. Nevertheless, this is not the case for a tangential $H$-field at such interfaces. Maxwell's equation $\vec{\nabla} \times \vec{H}=\vec{J}+$ $\partial \vec{D} / \partial t$ can be used to create an interfacial current density, $\vec{J}$. The high conductance of aluminum or gold permits the flow of the surface current. Using the charge conservation principle for this specific medium, we obtain $\nabla \overrightarrow{. J}+\partial \rho / \partial t=0$, where $\rho$ is the surface charge density. The motion of the conduction electrons corresponds to a surface current density $\vec{J}=q \vec{V}$ and the only source of electrical currents are bound charges, with a current density, $\overrightarrow{J_{\mathrm{b}}}$, of

$\overrightarrow{J_{\mathrm{b}}}=\frac{\partial \vec{P}}{\partial t}=-\mathrm{i} \omega \epsilon_{0}(\epsilon-1) \vec{E}$

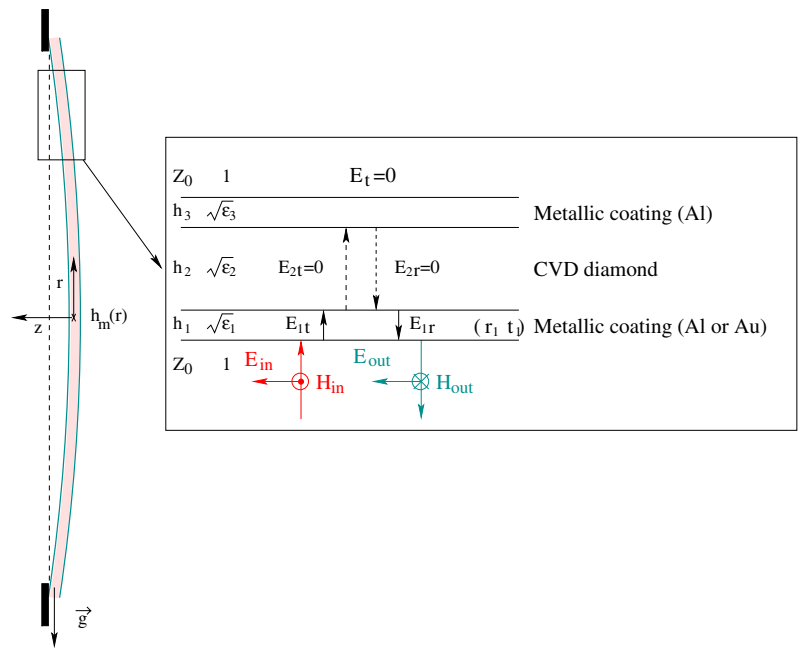

Fig. 2. Composition of the thin membrane and how the light from the laser interacts in its interior. The membrane consists of three films: (a) an aluminum coating of width $h_{1}$, (b) a CVD-diamond substrate of width $h_{2}$, and (c) an aluminum or gold coating, of width $h_{3}$. Left: the shape of the clamped-edge membrane, $h_{\mathrm{m}}(r)$, is only depending on $r$, and in the classical adaptive optics implementations, the gravity vector is vertical. Right: a linearly polarized plane wave $\left(E_{\text {in }}, H_{\text {in }}\right)$ is normally incident on the surface of a medium of complex dielectric constant $\epsilon_{1} . E_{1 \mathrm{t}}, E_{1 \mathrm{r}}$ are the electric fields inside the first reflective medium for, the transmitted and reflected waves respectively. For a good reflection of light with aluminum or gold, the thickness of the coating must be greater than $50 \mathrm{~nm}$ and the two electric fields inside with medium will be strongly attenuated. In this case, $E_{2 \mathrm{t}}, E_{2 \mathrm{r}}, E_{\mathrm{t}}$ inside and outside the CVD-diamond will be equal to zero. $Z_{0}=\sqrt{\mu_{0} / \epsilon_{0}}$ is the free-space impedance and the $H$-field amplitude will be always $H_{n}=\sqrt{\epsilon} E_{n} / Z_{0}$ depending on the relative permittivity $\epsilon$ of the medium.

The $H$-field of the electromagnetic wave will create a force on the bound current, which, according to the Lorentz law, will be

$F=1 / 2.2\left(\overrightarrow{J_{\mathrm{b}}} \times \overrightarrow{B^{*}}\right)$

where $\mathfrak{R}$ is the real part of the vectorial product between the bound current density, $\overrightarrow{J_{\mathrm{b}}}$, and the magnetic field $\vec{B}$.

\subsection{Forces on a homogeneous membrane with clamped edge}

In the vacuum, the amplitude of the $H$-field of the electromagnetic wave is proportional to the $E$-field $\left(H_{0}=E_{0} / Z_{0}\right)$ with $Z_{0}=\sqrt{\mu_{0} / \epsilon_{0}}$ being the free-space impedance. In a dielectric medium with a relative permittivity $\epsilon_{n}$ this relation becomes $H_{n}=\sqrt{\epsilon_{n}} E_{n} / Z_{0}$.

As shown in Fig. 2, the membrane is illuminated at a normal incidence, the complex reflection and transmission coefficients are denoted by $r_{1}$ and $t_{1}$ respectively, and the absorption coefficient is $A_{1}$ (see Table 1 ). The counter-propagating beams within the first aluminum coating have an $E$-field amplitude $E_{1 \mathrm{t}}$ and $E_{1 \mathrm{r}}$ and the total field distribution is given by

$E_{x}=E_{1 \mathrm{t}} \mathrm{e}^{\mathrm{i} \Gamma z}+E_{1 \mathrm{r}} \mathrm{e}^{-\mathrm{i} \Gamma z}$

$H_{y}=\left(\sqrt{\epsilon_{1}} E_{1 \mathrm{t}} / Z_{0}\right) \mathrm{e}^{\mathrm{i} \Gamma z}-\left(\sqrt{\epsilon_{1}} E_{1 \mathrm{r}} / Z_{0}\right) \mathrm{e}^{-\mathrm{i} \Gamma z}$ 
Table 1. Parameters used for the reflection coating.

\begin{tabular}{lcccccc}
\hline \hline $\begin{array}{l}\text { Material } \\
\text { coating }\end{array}$ & $\begin{array}{c}\lambda \\
(\mu \mathrm{m})\end{array}$ & $\begin{array}{c}\sqrt{\epsilon_{1}} \\
n+\mathrm{i} \kappa\end{array}$ & $\begin{array}{c}\text { Thickness } \\
(\mathrm{nm})\end{array}$ & $\begin{array}{c}\left|r_{1}\right|^{2} \\
\%\end{array}$ & $\begin{array}{c}\left|t_{1}\right|^{2} \\
\%\end{array}$ & $\begin{array}{c}A_{1} \\
\%\end{array}$ \\
\hline $\mathrm{Al}^{1}$ & 0.98 & $1.468+\mathrm{i} 9.22$ & 100 & 93.83 & 0 & 6.17 \\
$\mathrm{Au}^{2}$ & 0.98 & $0.248+\mathrm{i} 6.67$ & 60 & 97.85 & 0 & 2.15 \\
\hline
\end{tabular}

Notes. (1) Mearini (1992), Barron (2005), Bass et al. (2009); (2) Bass et al. (2009).

$$
\begin{aligned}
& E_{1 \mathrm{t}}=2 E_{\mathrm{in}} /\left((1+\rho)+\sqrt{\epsilon_{1}}(1-\rho)\right) \quad E_{1 \mathrm{r}}=\rho E_{1 \mathrm{t}} \\
& \Gamma=2 \pi \sqrt{\epsilon_{1}} / \lambda \quad \rho=\left(\sqrt{\epsilon_{1}}-1\right) /\left(\sqrt{\epsilon_{1}}+1\right) \mathrm{e}^{\mathrm{i} 2 \Gamma h_{1}} \\
& r_{1}=\frac{\rho-\left(\left(\sqrt{\epsilon_{1}}-1\right) /\left(\sqrt{\epsilon_{1}}+1\right)\right)}{1-\rho\left(\left(\sqrt{\epsilon_{1}}-1\right) /\left(\sqrt{\epsilon_{1}}+1\right)\right)} \\
& t_{1}=\frac{4 \sqrt{\epsilon_{1}}}{\left(\sqrt{\epsilon_{1}}+1\right)^{2} \mathrm{e}^{-\mathrm{i} \Gamma h_{1}}-\left(\sqrt{\epsilon_{1}}-1\right)^{2} \mathrm{e}^{\mathrm{i} \Gamma h_{1}}} \\
& \left|r_{1}\right|^{2}+\left|t_{1}\right|^{2}=1+A_{1} \quad A_{1}>0 .
\end{aligned}
$$

Therefore, the radiation pressure force, $F_{z}$, becomes

$$
F_{z}=\epsilon_{0}\left(\left|r_{1}\right|^{2}-A_{1}\right) E_{\text {in }}^{2}
$$

\section{Optical simulation}

This section presents a complete analytical simulation of the electromagnetic field due to the VCSEL's array on the membrane. The VCSEL array is composed of $n_{x} \times n_{y}$ lasers. Each laser monomode diode creates a Gaussian illumination on the microlens array. The proposed optical system propagates this laser mode onto the membrane with the same shape. Nevertheless, in the classical micro lens array, the Fresnel diffraction pattern between the lenses sets a limitation on their filling factor. Indeed, it is currently impossible to manufacture lenselet arrays with $100 \%$ of filling factor (it is usually around 99\%). If the lens pitch in the $x$ and $y$ directions are $50 \mu \mathrm{m}$, the diffractive zone is $\approx 0.5 \mu \mathrm{m}$. All lens boundaries create Fresnel diffraction is called a waffle effect.

The entrance amplitude light field in each microlens is considered Gaussian with the following definition:

$U_{i, j}^{\mathrm{L}}=\sqrt{I_{i, j}^{\mathrm{L}}} \mathrm{e}^{-\left(\left(x-x_{i, j}\right)^{2}+\left(y-y_{i, j}\right)^{2}\right) /\left(4\left(\sigma_{g x}^{2}+\sigma_{g y}^{2}\right)\right)}$,

where $I_{i, j}^{\mathrm{L}}$ is the intensity of the laser $(i, j)$, and $x_{i, j}, y_{i, j}$ are the cartesian coordinates of the beam center for the laser $(i, j)$. The 4 factor on the beam width in amplitude corresponds to a classical Gaussian distribution beam (with the factor of 2 in the denominator) for the intensity laser,

$\left(x_{i, j}, y_{i, j}\right)=\left(\frac{(2 i-1) \Lambda_{x}}{2}, \frac{(2 j-1) \Lambda_{y}}{2}\right) \quad i \in\left\{0, n_{x}\right\} \quad j \in\left\{0, n_{y}\right\}$.

Finally, we assume that each Gaussian is symmetric, with $\sigma_{\mathrm{g} x}=$ $\sigma_{\mathrm{g} y}$, although this will not be the case if the laser diode presents beam astigmatism.

Now, we calculate the Fresnel diffraction caused by the imperfect filling factor on the square array of laser diodes. The array pitch is set by the previously defined $\Lambda_{x}$ and $\Lambda_{y}$ distances.

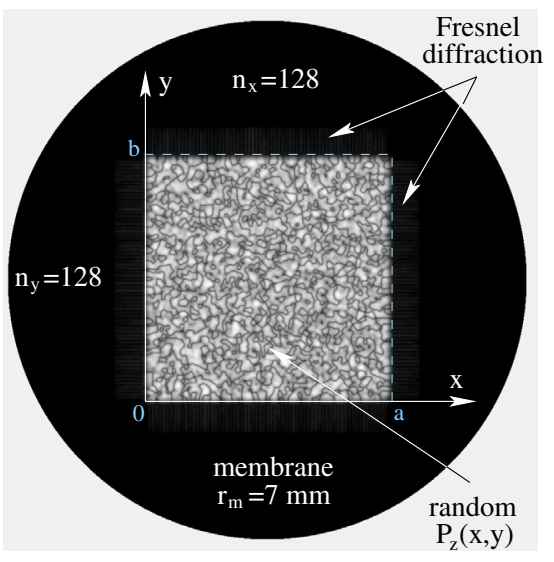

Fig. 3. Numerical simulation of the illuminated field $P_{z}$ obtained by the proposed VCSEL array $(128 \times 128$, pitch of $50 \mu \mathrm{m})$. We chose a circular clamped membrane of $r_{\mathrm{m}}=7 \mathrm{~mm}$ (circle limit). Our simulation considers a random wavefront error of 0.1 wave rms and includes the Fresnel propagation. To enhance the last effect in the image, the brightness scale is not linear $\left(|I|^{0.25}\right)$.

$F_{x}$ and $F_{y}$ are the filling factors in the cartesian coordinates, with $F_{x}=0.99 \Lambda_{x}$ and $F_{y}=0.99 \Lambda_{y}$.

The entire demonstration is given in appendix A and the results are presented in Fig. 3.

For $z=2 \mathrm{~cm}$ and $\lambda=0.98 \mu \mathrm{m}$, all Fresnel numbers are $\ll 1$ (Fraunhofer diffraction mode). A numerical calculation shows that $U_{\mathrm{Fr}}<10^{-4} U_{\mathrm{g}}$, which means that the waffle effect caused by the imperfect filling factor seems to be very low. The total illuminated laser field on the membrane can be considered continuous. The membrane mechanical deformation with $n_{x} \times n_{y}$ actuators will be modelled as a single, large actuator. The full analytical development is the scope of the following section.

\section{Modeling the membrane deformation $w$}

\subsection{Equation of motion for a membrane in cartesian coordinates}

Let $w=w(x, y, t)$ be the mechanical membrane deformation, and $P_{z}=P_{z}(x, y, t)$ the radiative pressure given by the VCSEL array. The full dynamical mechanical deformation is

$$
\begin{aligned}
& -\frac{1}{a b}\left[\bar{\rho} h \frac{\partial^{2} w}{\partial t^{2}}+\zeta \frac{\partial w}{\partial t}+K w\right] \\
& \quad+\left[D \nabla^{4} w-\frac{P_{t}}{a b} \nabla^{2} w-\frac{h}{a b} G\left(\phi_{s}, \sigma\right)\right]=P_{z}(x, y, t) .
\end{aligned}
$$

Assuming that the temporal part of the radiation pressure is equal to the membrane deformation, the the solution to Eq. (17) can be decomposed into a spatial and temporal component as follows:

$$
w=w(x, y) w(t) \quad \text { and } \quad P_{z}=P_{z}(x, y) w(t) .
$$

Plugging Eqs. (18) into (17) yields the temporal and spatial equations as follows:

$$
\begin{aligned}
& \bar{\rho} h \frac{\partial^{2} w(t)}{\partial t^{2}}+\zeta \frac{\partial w(t)}{\partial t}+\left(K+\frac{P_{z}(x, y) a b}{w(x, y)}\right) w(t)=0 \\
& D \nabla^{4} w(x, y)-\frac{P_{t}}{a b} \nabla^{2} w(x, y)-\frac{h}{a b} G\left(\phi_{s}(x, y), \sigma\right)=P_{z}(x, y) .
\end{aligned}
$$

Details about each of the terms the temporal and spatial equations consist of will be provided in the following sections. 
Table 2. Material properties.

\begin{tabular}{|c|c|c|c|c|c|}
\hline Material & $\begin{array}{l}\text { Young's modulus } \\
\text { (GPa) }\end{array}$ & $\begin{array}{l}\text { Stress } \sigma \\
(\mathrm{MPa})\end{array}$ & $\begin{array}{l}\text { Poisson's } \\
\text { ratio }\end{array}$ & $\begin{array}{l}\text { Density } \\
\left(\mathrm{g} / \mathrm{cm}^{3}\right)\end{array}$ & $\begin{array}{l}\text { Roughness } \\
\text { (nm) }\end{array}$ \\
\hline CVD diamond ${ }^{1}$ & $610-1140$ & 0 to 100 & 0.1 & 3.51 & $1-3$ \\
\hline $\mathrm{Al}^{2}$ & $65-80$ & 20 & 0.35 & 2.7 & $1-10$ \\
\hline $\mathrm{Al} 0.5 \% \mathrm{Cu}^{3}$ & $60-120$ & -20 & 0.33 & 2.7 & 1 \\
\hline $\mathrm{Au}^{4}$ & 70 & -180 to 77 & 0.44 & 19.3 & $2-3^{\dagger}$ \\
\hline
\end{tabular}

Notes. ${ }^{(\dagger)}$ Direct AFM measurements of gold coated mirror. ${ }^{(1)}$ Hess (2012) for a complete review of CVD-diamond mechanical properties. (2) Mearini (1992). ${ }^{(3)}$ Barron (2005). ${ }^{(4)}$ Pandey et al. (2009), Salvadori et al. (2003), Thornell et al. (1999).

Appendix B gives static solutions in the Fourier plane for the linear model. These solutions are needed to enable the numerical simulations of the membrane deformation.

\subsection{Numerical calculation: the Galerkin method}

Prior to the development of the finite element method (FEM), there existed an approximation and recursive technique to solve differential equations, known as the Galerkin method (Galerkin 1915). The basic idea of this technique is to use the Fourier development given by Eq. (B.3) with $W_{n, m}$ as unknown parameters to approximate the solution iteratively. For more information concerning this method see Dubey (2005), and Kerrour \& Hobar (2006). The Galerkin method is also a method of weighted residuals, where over the interior and boundary an appropriate function is set to zero. Now, we carefully explain the calculation to obtain de radiation pressure $P_{z}(x, y)$ and the membrane deformation $w(x, y)$ (the final wavefront).

We define the weighted residuals $R(x, y)$ as

$$
\begin{aligned}
& R(x, y)=D \nabla^{4} w(x, y)-\frac{P_{t}}{a b} \nabla^{2} w(x, y)-P_{z}(x, y) \\
& \int_{0}^{a} \int_{0}^{b} R(x, y) S_{m}(x) S_{n}(y) \mathrm{d} x \mathrm{~d} y=0,
\end{aligned}
$$

where $w(x, y)$ is the solution on the DM array:

$$
\begin{aligned}
R(x, y)= & \sum_{m^{\prime}=1}^{n_{y}} \sum_{n^{\prime}=1}^{n_{x}} W_{m^{\prime}, n^{\prime}}\left(D \nabla^{4}\left[S_{m^{\prime}}(x) S_{n^{\prime}}(y)\right]\right) \\
& -\sum_{m^{\prime}=1}^{n_{y}} \sum_{n^{\prime}=1}^{n_{x}} W_{m^{\prime}, n^{\prime}}\left(\frac{P_{t}}{a b} \nabla^{2}\left[S_{m^{\prime}}(x) S_{n^{\prime}}(y)\right]\right) \\
& -\frac{4}{a b} \int_{0}^{a} \int_{0}^{b} P_{z}(x, y) S_{m}(x) S_{n}(y) \mathrm{d} x \mathrm{~d} y .
\end{aligned}
$$

After some calculations, Eq. (23) gives

$$
\begin{aligned}
& \sum_{m=1}^{n_{x}} \sum_{n=1}^{n_{y}} W_{m, n}\left[D\left(\left(\frac{m \pi}{a}\right)^{2}+\left(\frac{n \pi}{b}\right)^{2}\right)^{2}-\frac{P_{t}}{a b}\left(\left(\frac{m \pi}{a}\right)^{2}+\left(\frac{n \pi}{b}\right)^{2}\right)\right]= \\
& \frac{4}{a b} \int_{0}^{a} \int_{0}^{b}\left[\frac{1}{c} \frac{\left(\left|r_{1}\right|^{2}-A_{1}\right)}{a b} U_{\mathrm{f}}(x, y) U_{\mathrm{f}}^{*}(x, y)\right] S_{m}(x) S_{n}(y) \mathrm{d} x, \mathrm{~d} y
\end{aligned}
$$

where $c$ is the speed of light in the vacuum. The total illuminated laser field is $U_{\mathrm{f}}=U_{\mathrm{g}}+U_{\mathrm{Fr}} \approx U_{\mathrm{g}}$. This is because, as we previously calculated, the Fresnel propagation is negligible, with $U_{\mathrm{Fr}}<10^{-4} U_{\mathrm{g}}$,

$U_{\mathrm{f}}\left(x_{m, n}, y_{m, n}\right) U_{\mathrm{f}}^{*}\left(x_{m, n}, y_{m, n}\right) \approx \sum_{m=1}^{n_{x}} \sum_{n=1}^{n_{y}} U_{m, n}^{\mathrm{L}} U_{m, n}^{L *}$.
$U_{m, n}^{\mathrm{L}} U_{m, n}^{L *}$ is the influence function of actuator $m, n$, and $\left(x_{m, n}, y_{m, n}\right)$ are the cartesian coordinates of the actuator center (see Eq. (15)). However, the final shape of the mirror surface depends on the positions of the neighbor actuators. The two matrixes $W_{m, n}$ and $I_{z, m, n}$ are not diagonals. Solving this linear system allows us to fully characterize this influence function.

The membrane deformation linear model, presented in this section, is the first approximation to retrieve the relationship between $w(x, y)$ and $P_{z}(x, y)$. However, two corrections must be applied on this model:

- because the membrane is a multi-layer system, its mechanical properties must be corrected for;

- the deformation $w(x, y)$ becomes nonlinear when the amplitude is larger than the membrane thickness. Therefore a nonlinear model that includes an Airy stress function is preferable to obtain a better evaluation of membrane deformation amplitude $w(x, y)$.

\subsection{Multi-element membrane}

The mechanical properties of a multi-layer system differ from a single-layer homogenous membrane. Because the Poisson's ratios differ for diamond/aluminum, there will be a change in the length of the lines that are parallel to the lower material (Al) due to an in-plane strain $\epsilon_{z}$. An equivalent single-material approach, Myers et al. (2006), can be used for this study if

$D \rightarrow D_{\mathrm{e}} \quad v \rightarrow v_{\mathrm{e}} \quad E \rightarrow E_{\mathrm{e}}$,

where $D_{\mathrm{e}}, v_{\mathrm{e}}, E_{\mathrm{e}}$ are the equivalent mechanical properties of the proposed structure. Our model is composed of four layers of different materials. The top layers are considered as one bi-material layer (Al $100 \mathrm{~nm} /$ diamond $500 \mathrm{~nm}$ ) and the bottom one as well (diamond $500 \mathrm{~nm} / \mathrm{Al} 100 \mathrm{~nm}$ or diamond $500 \mathrm{~nm} / \mathrm{Au} 60 \mathrm{~nm}$ ). Some mechanical properties of these materials are summarized in Table 2. The mathematical expressions of $D_{\mathrm{e}}, v_{\mathrm{e}}, E_{\mathrm{e}}$ are given in Appendix C.

For the layer combination $\mathrm{Al} / \mathrm{Au}$ we chose an average Young's modulus of $70 \mathrm{GPa}$ with a Poisson's ratio of 0.33 and 0.44 , respectively. Concerning the Stress coefficient $\sigma$, we chose the conservative value of $20 \mathrm{MPa}$. A coating optimization of the $\mathrm{Al} /$ diamond/Au system can decrease near the zero value the stress coefficient.

\subsection{Deflection of the membrane}

The deflection of a circular clamped membrane is given by Sheplak \& Seiner (1999):

$h_{m}(r)=\frac{\Delta P r_{m}^{4}}{T_{m}^{2} D_{\mathrm{e}}}\left(\frac{I_{0}\left(T_{m} r / r_{m}\right)-I_{0}\left(T_{m}\right)}{2 T_{m} I_{1}\left(T_{m}\right)}+\frac{r_{m}^{2}-r^{2}}{4 r_{m}^{2}}\right)$ 
Table 3. Parameters used for the composite membrane.

\begin{tabular}{lccccc}
\hline \hline $\begin{array}{l}\text { Membrane } \\
\text { structure }\end{array}$ & $\begin{array}{c}\text { Stiffness } \\
(\mathrm{N} / \mathrm{m})\end{array}$ & $\begin{array}{c}\text { Young's modulus } \\
(\mathrm{GPa})\end{array}$ & $\begin{array}{c}\text { Stress } \sigma \\
(\mathrm{MPa})\end{array}$ & $\begin{array}{c}\text { Poisson's } \\
\text { ratio }\end{array}$ & $\begin{array}{c}\text { Density } \bar{\rho} \\
\mathrm{g} / \mathrm{cm}^{3}\end{array}$ \\
\hline $\mathrm{Al} /$ diamond/Al & $4.984 \times 10^{-8}$ & 345 & 20 & 0.07 & 3.375 \\
$\mathrm{Al} /$ diamond/Au & $4.985 \times 10^{-8}$ & 375 & 20 & 0.07 & 4.257 \\
\hline
\end{tabular}

$T_{m}=\frac{r_{m}}{h} \sqrt{\frac{12\left(1-v_{\mathrm{e}}^{2}\right) \sigma}{E_{\mathrm{e}}}}$,

where $\Delta P$ is differential pressure, $T_{m}$ is a tension parameter due to the residual stress $\sigma$ in the membrane, $I_{0}\left(T_{m}\right)$ and $I_{1}\left(T_{m}\right)$ are modified Bessel function on the order of 0 and 1 respectively. The maximum deflection of the membrane is given for $r=0$,

$$
h_{\mathrm{m}}(0)=\Delta P(\mathrm{~Pa}) 500 \mathrm{~nm} \quad T_{\mathrm{m}}=152.287 \quad \frac{I_{0}\left(T_{\mathrm{m}}\right)}{I_{1}\left(T_{\mathrm{m}}\right)}=1.00328 .
$$

We notice that the deformation memory will be very low for diamond material because this effect occurs only on the elasticity deformation region ( $>95 \mathrm{GPa}$ ) and diamond has the highest elastic moduli and fracture strength of any known material. In the real world, the membrane breaks with the cleavage process before non-reversible deformation. For cubic materials, the crack occurs around $E / 10 \approx 110 \mathrm{GPa}$ (Hess 2012).

\subsection{Nonlinear model for the static solutions}

Our surface deflection amplitude can be more than the membrane thickness. In this case, the vertical deflections are accompanied by stretching of the mirror surface. The stretching is a nonlinear process that causes the mirror layer to increase its load-carrying capabilities. To include this nonlinear stretching of the membrane into our model the plate equation must modified as

$D_{\mathrm{e}} \nabla^{4} w-\frac{P_{t}}{a b} \nabla^{2} w-\frac{h}{a b} G\left(\phi_{s}, \sigma\right)=P_{z}(x, y) \quad h=h_{1}+h_{2}+h_{3}$.

The complete evaluation of this equation using the same Galerkin method as the linear model in Appendix B is given in Appendix D.

\section{DM: the spatial response}

Using the methods describe above, we simulated our $128 \times$ 128 DM. First, we used the linear model with the membrane composite stiffness $D_{\mathrm{e}}$ to estimate the deformation function $w^{\mathrm{L}}(x, y)$. Equation (24) can be put into matrix form: $A_{w} W_{m, n}^{\mathrm{L}}=I_{z, m, n}$.

Second, we used Eq. (D.17) to estimate the Airy stress function with the linear solution of the deformation function $w^{\mathrm{L}}(x, y)$. We calculated the new deflection function $w^{\mathrm{NL}}(x, y)$ using the nonlinear model Eq. (D.18). Finally, to increase the precision of the $w(x, y)$ result, we repeated the two last processes until the desired relative error was achieved.

\subsection{Direct evaluation}

For our study, we needed to obtain the input laser field for a known membrane deformation. In this context, this is not necessary to resolve the system of equations.

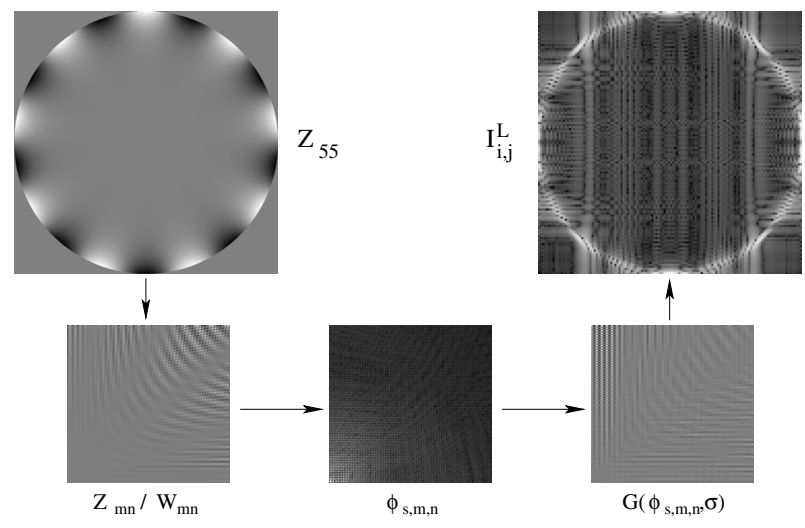

Fig. 4. Example of the nonlinear simulation on Zernike 55. The projection of $Z_{55}$ in the Fourier plane gives the $Z_{m, n} / W_{m, n}$ coefficients. The Airy-type stress function $\phi_{s, m, n}$ is evaluated by the $W_{m, n}$ coupling (see Eq. (D.17)). The nonlinear part of the Eq. (29) $\left(G\left(\phi_{s}, \sigma\right)\right)$ is calculated and the $I_{z, m, n}$ is directly obtained. A deconvolution by the Gaussian laser beam distribution $\left(E_{\mathrm{g} x y}\right)$ and the final projection on the DM plane gives $I_{i, j}^{\mathrm{L}}$. All images are given in linear brightness except for $\phi_{s, m, n}$ where the intensity is power of 0.1 .

The laser intensity was evaluated directly using the nonlinear model (Eq. (D.18)). First of all, we assumed that the final membrane deformation $w(x, y)$ is a pure Zernike polynomial $Z_{p}$. Using Eq. (B.7), we numerically projected the Zernike polynomial $Z_{p}$ on the $S_{m}(x) / S_{n}(y)$ Fourier basis, we obtained the $Z_{m, n}$ coefficients as $W_{m, n}$. As we explain in the following scheme, we evaluated the nonlinear function $G$ and calculated the input laser flux $I_{i, j}^{\mathrm{L}}$,

$Z_{p} \longrightarrow Z_{m, n} \longrightarrow\left[\phi_{s, m, n} / G(0, \sigma)\right]_{\mathrm{NL}} \longrightarrow\left[G\left(\phi_{s}, \sigma\right)\right]_{\mathrm{NL}} \longrightarrow I_{z, m, n}$.

Using the $I_{z, m, n}$ function evaluation in Appendix E for a purely Gaussian laser field (without the Fresnel propagation), we obtain $I_{z, m, n} \rightarrow\left(E_{\mathrm{g} x y}\right) \rightarrow I_{m, n}^{\mathrm{L}} \rightarrow I_{i, j}^{\mathrm{L}}$.

An example using an input Zernike $\left(Z_{55}\right)$ in the pupil plane is presented in Fig. 4.

\subsection{Positive vs. negative correction}

Our proposed optical MEMS using the radiation pressure as actuator performs wavefront deflection in the same way (always the positive values). Negative wavefront values are also allowed but we need to add an input curvature as a defocus $\propto 4 r^{2} / a^{2}$ on the membrane. The input wavefront $Z_{p}$ is changed to $Z_{p}^{ \pm}$as

$Z_{p}^{ \pm}(2 r / a, \theta)=f_{w} \frac{\sqrt{3}}{2} Z_{4}(2 r / a)+\sum_{p=1}^{p_{\max }} \pm \beta_{p} Z_{p}(2 r / a, \theta)$,

where $Z_{p}^{ \pm}(2 r / a, \theta)$ is the desired entrance wavefront (here a sum of simple Zernike polynomials) in the $r, \theta$ polar coordinate basis, $\pm \beta_{p}$ are the Zernike weight coefficients, and $f_{w}$ is the 
Table 4. Cavity depth and damping coefficient for $\mathrm{Al} /$ diamond/Au membrane.

\begin{tabular}{lcccccc}
\hline \hline Material & $\begin{array}{c}\text { Density } \\
\rho_{0}\left(\mathrm{~kg} / \mathrm{m}^{3}\right)\end{array}$ & $\begin{array}{c}\text { Sound velocity } \\
c_{a}(\mathrm{~m} / \mathrm{s})\end{array}$ & $\begin{array}{c}\text { Damping } \\
\zeta\end{array}$ & $\begin{array}{c}\text { Cavity depth } \\
L_{\text {cav }}(\mathrm{mm})\end{array}$ & $\begin{array}{c}\text { Stiffness } \\
\mathrm{K}\left(\mathrm{N} / \mathrm{m}^{3}\right)\end{array}$ & $\begin{array}{c}\omega_{0} / 2 \pi \\
(\mathrm{KHz})\end{array}$ \\
\hline air & 1.293 & 343 & 0.02 & 1 & $>1.5 \times 10^{8}$ & 27.9 \\
aerogel $^{1}$ & $50-300$ & $\approx 100$ & $5-100$ & 1 & $7 \times 10^{6}$ & 6.0 \\
\hline
\end{tabular}

Notes. ${ }^{(1)}$ Zimmermann et al. (1995), Daughton et al. (2003).

level of membrane defocus. We present the corrected wavefront as a finite sum of Zernikes between $p=1$ to $p_{\max } \approx 10000$ for $128 \times 128$ actuators.

Under these conditions, the dynamic of the optical MEMS is decreased by a factor of about 2 to perform both positive and negative wavefront corrections. The maximum deflection is calculated according to our previous nonlinear model (see Sect. D), representing the full dynamic of the proposed architecture (i.e. $1 \mu \mathrm{m}= \pm 500 \mathrm{~nm}$ ).

\section{DM: dynamical modeling}

We notice that the spatial nonlinear equation (see Eq. (29)) is given in cartesian coordinates because of the square shape of the $n_{x} \times n_{y}$ actuators. The radiation pressure acts as forced vibration on a clamped circular membrane and we coupled the possible vibration effect of actuators with some natural modes of vibration of the circular membrane.

\subsection{The DM frequency response}

To properly calculate the coupling parameter, we write the spatial and temporal equations in the following way.

$\nabla^{4} w(x, y)-\frac{P_{t}}{D_{\mathrm{e}} a b} \nabla^{2} w(x, y)-\frac{h}{D_{\mathrm{e}} a b} G\left(\phi_{s}(x, y), \sigma\right)-\lambda_{m}^{4} w(x, y)=0$

$\frac{\partial^{2} w(t)}{\partial t^{2}}+\frac{\zeta}{\bar{\rho} h} \frac{\partial w(t)}{\partial t}+\left(\omega_{0}^{2}+\omega^{2}\right) w(t)=0$

$\lambda_{m}^{4}=\frac{P_{z}}{w D_{\mathrm{e}}} \quad \omega^{2}=\frac{P_{z}}{w} \frac{a b}{\bar{\rho} h} \quad \omega_{0}^{2}=\frac{K}{\bar{\rho} h}$

- where $\bar{\rho} h$ is the main dynamical coefficient proportional to the mass of the membrane;

- $\zeta$ is the damping coefficient (including air viscous damping);

- $K+P_{z}(x, y) a b / w(x, y)$ is the stiffness coefficient of the proposed system (including the air stiffness $K$ inside a cavity).

The resolution of the temporal equation gives

$w(t)=\mathrm{e}^{\frac{-t \zeta}{2 \rho h}}\left(\frac{1}{C_{\zeta}} \sinh \left(\frac{-t}{2 \rho h} C_{\zeta}\right)+\cosh \left(\frac{-t}{2 \rho h} C_{\zeta}\right)\right)$

$C_{\zeta}=\sqrt{\zeta^{2}-4 \bar{\rho}^{2} h^{2}\left(\omega_{0}^{2}+\omega^{2}\right)}$.

\subsection{Resonance frequencies calculation for a circular clamped membrane}

In the previous analysis, the membrane temporal motion was not governed by resonance frequencies. Indeed, the $P_{z} / w$ parameter
Table 5. Some $f_{\mathrm{c}, m, n}=\omega_{c}^{2} / 2 \pi$ values in $\mathrm{Hz}$ for the free vibration of the $\mathrm{Al} /$ diamond/Au membrane.

\begin{tabular}{ccccc}
\hline \hline$m$ & \multicolumn{4}{c}{$n$} \\
\hline & 1 & 2 & 3 & 4 \\
0 & 105 & 219 & 360 & 527 \\
1 & 410 & 628 & 873 & 1145 \\
2 & 919 & 1239 & 1587 & 1964 \\
3 & 1632 & 2054 & 2505 & 2984 \\
4 & 2549 & 3073 & 3626 & 4208 \\
\hline
\end{tabular}

is not a modal function of a circular clamped membrane. If we are interested in the effect of resonance frequencies on the membrane dynamic, we need to perform a modal analysis for a circular clamped membrane in polar coordinates (see Mehta 2009), which gives

$$
\begin{aligned}
& w(r, \theta) \propto\left(J_{m}\left(\lambda_{c} r\right) I_{m}\left(\lambda_{c} r_{m}\right)-J_{m}\left(\lambda_{c} r_{m}\right) I_{m}\left(\lambda_{c} r\right)\right)(\cos (m \theta) \\
& \quad+\sin (m \theta)) .
\end{aligned}
$$

The frequency equation with infinitely many solutions for each value of $m$ is

$$
\begin{aligned}
& {\left[\frac{\partial J_{m}\left(\lambda_{c} r\right)}{\partial r} I_{m}\left(\lambda_{c} r_{m}\right)-J_{m}\left(\lambda_{c} r_{m}\right) \frac{\partial I_{m}\left(\lambda_{\mathrm{c}} r\right)}{\partial r}\right]_{r=r_{m}}=0} \\
& J_{m-1}\left(\lambda_{\mathrm{c}, m, n} r_{m}\right) I_{m}\left(\lambda_{\mathrm{c}, m, n} r_{m}\right)-J_{m}\left(\lambda_{\mathrm{c}, m, n} r_{m}\right) I_{m-1}\left(\lambda_{\mathrm{c}, m, n} r_{m}\right)=0 .
\end{aligned}
$$

Each Bessel function also possesses $n$ roots (nodes), therefore $\lambda_{\mathrm{c}}$ is a function of the $m$ and $n$ coefficients. We notice that these two parameters $n, m$ are not the same physical meaning as the previous Fourier analysis for spatial solution. The values of resonance frequencies are given by

$\omega_{c}^{2}=\frac{\lambda_{\mathrm{c}, m, n}^{4}}{r_{m}^{4}} \frac{D_{\mathrm{e}}}{\bar{\rho} h}$.

We also point out that the first frequency for an $\mathrm{Al} /$ diamond/Al membrane is $f_{c, 0,1}=116 \mathrm{~Hz}$ compared to the $\mathrm{Al} /$ diamond $/ \mathrm{Au}$ membrane, where $f_{c, 0,1}=105 \mathrm{~Hz}$ (see Table 5).

\subsection{The modal decomposition by a modified Hankel transform}

We previously calculated the temporal response of the membrane as a function of $P z(x, y) / w(x, y)$ (see Eq. (32)). The important question is to know whether there exists natural resonance for a fixed value of wavefront correction $w(x, y)$ or not. For a membrane deflection $w=w(r, \theta)$ (Eq. (34)), we propose to 
calculate the resonance coefficients $\alpha_{\mathrm{c}, m, n}$ using a modified version of the Hankel transform,

$$
\begin{aligned}
& H_{\lambda_{\mathrm{c}}, m, n}^{ \pm}(w)=\int_{0}^{2 \pi} \int_{0}^{1} r w J_{m}\left(\lambda_{\mathrm{c}, m, n} r_{m} r\right) \mathrm{e}^{ \pm \mathrm{i} \theta / 2} \mathrm{~d} r \mathrm{~d} \theta \\
& \int_{0}^{2 \pi}(\cos (m \theta)+\sin (m \theta)) \mathrm{e}^{ \pm \mathrm{i} \theta / 2} \mathrm{~d} \theta=\frac{2 m \mp i}{\left(m+\frac{1}{2}\right)\left(m-\frac{1}{2}\right)} \\
& \int_{0}^{1} r J_{m}\left(\lambda_{\mathrm{c}, m, n} r_{m} r\right)^{2} \mathrm{~d} r=\frac{1}{2} J_{m}\left(\lambda_{\mathrm{c}, m, n} r_{m}\right)^{2} \\
& \int_{0}^{1} r I_{m}\left(\lambda_{\mathrm{c}, m, n} r_{m} r\right)^{2} \mathrm{~d} r=\frac{1}{2} I_{m}\left(\lambda_{\mathrm{c}, m, n} r_{m}\right)^{2} \\
& H_{\lambda_{c}, m, n}^{ \pm}(w)=\sum_{m, n} \alpha_{\mathrm{c}, m, n} \frac{2 m \mp i}{2\left(m+\frac{1}{2}\right)\left(m-\frac{1}{2}\right)} D_{\mathrm{c}, m, n}^{I J} \\
& D_{\mathrm{c}, m, n}^{I J}=\left(I_{m}\left(\lambda_{\mathrm{c}, m, n} r_{m}\right) J_{m}\left(\lambda_{\mathrm{c}, m, n} r_{m}\right)^{2}-J_{m}\left(\lambda_{\mathrm{c}, m, n} r_{m}\right) I_{m}\left(\lambda_{\mathrm{c}, m, n} r_{m}\right)^{2}\right) .
\end{aligned}
$$

We previously integrated the $w$ function on the $\theta$ with a modulation of $\mathrm{e}^{ \pm \mathrm{i} \theta / 2}$ as an azimuthally averaged function to perform the correct modal analysis.

The imaginary and real part correspond to the cos and the sin modulation of the wavefront. This calculation is the modal decomposition of the deflection function $w$ for $\cos / \sin$ modes.

If the input wavefront is a direct sum of Zernike polynomials (Nijboer-Zernike decomposition, see Magette 2010), the modal decomposition is more straightforward and we have

$$
\begin{aligned}
& w(r)=\sum_{m, n^{\prime}} R_{n^{\prime}}^{m}\left(r a / 2 r_{m}\right)\left(\beta_{c n^{\prime}}^{m} \cos (m \theta)+\beta_{s n^{\prime}}^{m} \sin (m \theta)\right) \\
& H_{\lambda_{c}, m, n, n^{\prime}}^{ \pm}(w)=\sum_{m, n^{\prime}}(-1)^{\frac{n^{\prime}-m}{2}} \frac{2 m \beta_{s n^{\prime}}^{m} \mp i \beta_{c n^{\prime}}^{m}}{\left(m+\frac{1}{2}\right)\left(m-\frac{1}{2}\right)} \cdot \frac{J_{n^{\prime}+1}\left(\lambda_{\mathrm{c}, m, n} a / 2\right)}{\left(\lambda_{\mathrm{c}, m, n} a / 2\right)}
\end{aligned}
$$

If we equalize Eqs. (42) with (44), we obtain the resonance frequencies $\alpha_{\mathrm{c}, m, n}$ as a function of Zernike coefficients $\beta_{n}^{m}$ as

$\alpha_{\mathrm{c}, m, n}=\sum_{n^{\prime}} \frac{(-1)^{\frac{n^{\prime}-m}{2}}\left(\beta_{s n^{\prime}}^{m}+\beta_{c n^{\prime}}^{m}\right) J_{n^{\prime}+1}\left(\lambda_{\mathrm{c}, m, n} a / 2\right)}{\left(\lambda_{\mathrm{c}, m, n} a / 2\right) D_{\mathrm{c}, m, n}^{I J}}$.

In this case, if a low-order Zernike polynomials have a larger amplitude with respect to each other, the deformable mirror will present overshooting behavior for some resonance frequencies given by our modal analysis.

\subsection{Passive vibration attenuation}

The DM system possesses a small cavity between the moving membrane and the polarizing optical system. This cavity can be open (pressure equilibrium) or closed. The cavity depth can be adjusted for the DM optimization. If we assume that this cavity depth was significantly smaller than the radius of the membrane, the ratio $L_{\text {cav }} / r_{m} \ll 1$. In this case, we can approximate the response of the air within the cavity as a distribution of springs and dampers acting on each differential element of the membrane (actuators). The cavity stiffness $K$ can be defined as

$K=\left(\rho_{0} c_{a}^{2}\right) / L_{\text {cav }}$.

The resonance frequencies and the cavity stiffness calculation show a useful frequency bandpass of about 1-10 KHz. Indeed, with an attenuation of $1 / f$ for natural resonance frequencies (see Table 5), and the Shannon theorem applied on the cavity stiffness frequency $\left(\omega_{0} / 2 \pi\right)$, the optimal frequency response of our optical DM is in the $\mathrm{KHz}$ range.

\section{Laser membrane heating: cooling parameters}

Under high-power laser irradiation, the mirror absorbs a small fraction of the incident light and is heated to temperatures much higher than those of the coating beneath it. The thermal degradation must be evaluated and minimized to prevent mechanical degradation or thermal shape deformation. A cooling mechanism overcomes this thermal effect.

We notice that the laser damage of a classical thin membrane is as low as $340 \mathrm{~W} / \mathrm{cm}^{2}$ for polyimide material, and $>100 \mathrm{KW} / \mathrm{cm}^{2}$ for silicon nitride.

It is important to understand the mechanisms of the thermally induced distortions. One possible explanation of the membrane distortion is that the high laser power creates a spatially varying temperature distribution, which may warp the surface. Another possible source of the thermally induced distortions is the differential thermal expansion of the membrane's material.

Concerning the silicon nitride material used for multilayer dielectric-coated deformable mirrors, Sinha et al. (2002) obtained a thermally induced wavefront distortion of 32.3 and $39.1 \mathrm{~nm}$ rms for an incident intensity of 140 and $212 \mathrm{~W} / \mathrm{cm}^{2}$, respectively, with a cw $1064 \mathrm{~nm}$ laser on a gold coated membrane. These values are very pessimistic regarding our freestanding reflective diamond membrane architecture. Indeed, the DM device used in Sinha et al. (2002) is a complex dielectric structure with silicon pillars, which explains the localization of the deformations by differential thermal expansion. A simple gold-coated silicon mirror presents no distortion for an incident intensity of $300 \mathrm{~W} / \mathrm{cm}^{2}$.

This last study must be compared with the present design, where the maximum incident laser intensity is $I_{\max }^{\mathrm{L}}=$ $970 \mathrm{~W} / \mathrm{cm}^{2}$ for $25 \mathrm{~mW}$ per diode. In our case, the estimated membrane conductivity is about $1588 \mathrm{~W} /(\mathrm{mK})$ for a gold-coated membrane and the thermal field distribution will be more uniform because the laser heating is efficiently conducted over the membrane surface because of the high conductivity of the materials $(1800 / 318 / 237 \mathrm{~W} /(\mathrm{mK})$ for diamond, gold, and aluminum, respectively).

In our irradiation condition, a thermally induced wavefront distortion of less than $20 \mathrm{~nm}$ rms seems to be achievable. The membrane cooling seems to be mandatory to perform high wavefront precision corrections (residual $<1 \mathrm{~nm}$ ) with a maximum absorbed laser power of $A_{1} I_{\max }^{\mathrm{L}} \approx 21 \mathrm{~W} / \mathrm{cm}^{2}$ for gold-back coating. Some studies that used silicon nitride material such as Morse et al. (2008) or Lee et al. (2006), show a more experimental analysis of deformable mirrors under high-power laser flux.

Another problem that occurs with laser heating is the mechanical thermal stress. Indeed, changes in temperature produce expansion or contraction, resulting in thermal strains and stress. For linear elastic materials, the thermal stress, $\sigma$, is governed by the following expression:

$\sigma(\Delta T)=\sigma+C_{\alpha} E_{\mathrm{e}} \Delta T$

where $C_{\alpha}=3.74 \times 10^{-6} \mathrm{~K}^{-1}$ is the thermal expansion of $\mathrm{Al} /$ diamond/Au membrane dominated by the two metallic layers. Indeed, the thermal expansion of diamond is only $1.18 \times$ $10^{-6} \mathrm{~K}^{-1}$ compared with 23.1 and $14.2 \times 10^{-6} \mathrm{~K}^{-1}$ for aluminum and gold, respectively.

\section{Optical cavity for high-power laser?}

The optical scheme presented in Fig. 1 permits using of a highreflectivity $(R>99.9 \%)$ cavity mirror to increase the laser power inside the cavity. This configuration could be used with a 


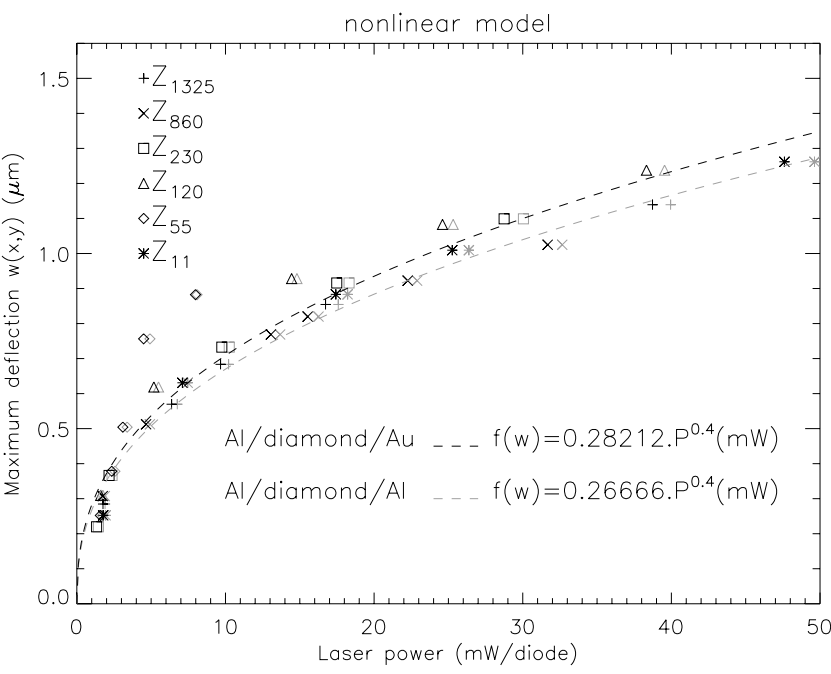

Fig. 5. Non linear simulation of the $w(x, y)$ maximum deflection with respect to the Zernike polynomial order. We present the deflection for two different membrane structures. The gold back-face coating shows a better dynamic than classical Al coating. A maximum deflection of $1 \mu \mathrm{m}$ with $\approx 25 \mathrm{~mW}$ per laser diode seems to be possible.

thicker membrane $(2-10 \mu \mathrm{m})$ on the linear mechanical deformation region (see Fig. 5 for the low-amplitude deformation range). Owing to the cooling process, to ensure a high wavefront precision correction, this configuration seems to be limited to increasing the wavefront dynamic of our proposed DM.

\section{Discussion}

We now discuss the practical aspects of the proposed DM concept. The very thin CVD-diamond membrane must be manufactured using nanocrystalline or ultra-nanocrystalline grown to ensure the best mechanical properties near the single diamond crystal with a low roughness (1-3 nm rms). Some laboratories and industries routinely manufacture such membranes for microoptical components such as MEMS.

The $\mathrm{Au} / \mathrm{Al}$ coating can be optimized to minimize the stress coefficient $\sigma$. Concerning the aluminum coating $\sigma=20 \mathrm{MPa}$ is generally obtained with classical sputtering system while for chromium-gold coating $\sigma=-20 \mathrm{MPa}$ can be reached after optimizing thermodynamical conditions.

Microlens arrays in fused silica with a very good wavefront error $(<\lambda / 20 \mathrm{rms}$ in the visible) and very accurate centering $(<0.5 \mu \mathrm{m})$ are commercially available, but a dedicated microoptical lens manufacturing can be achieved for each VCSELs laser (see Bardinal et al. 2011) to allow the best possible lens centering, with high-precision microelectronic technology.

Finally, the main challenge is the VCSELs array manufacturing, not for the near-infrared laser technology (micro-cavity with Bragg mirrors), but for the properties of the metal wires on the matrix-addressable array for a high-power system. Indeed, for a high-power laser system, where the overall laser conversion efficiency is between $35 \%$ to $50 \%$, the metal wire resistivity is an important limitation regarding the thermal management.

\section{Conclusion}

We showed the simulations of a new high-density deformable mirror using the VSCEL radiation pressure as actuators on a thin diamond membrane. A complete analysis with the nonlinear plate equation shows a dynamic of $\pm 0.5 \mu \mathrm{m}$ attainable with $25 \mathrm{~mW}$ per laser diode. Large array actuators (here $128 \times$ 128 actuators) allowed us to correct the first 10000 Zernike polynomials without segmentation effect.

The VCSEL array is modulated up to $1 \mathrm{GHz}$, but the temporal limitation response of the DM is mechanical because of the membrane response and its sufficient dampening. The dynamical analysis showed a possible use of the DM in 1 to $10 \mathrm{KHz}$. In theses cases, our proposed DM (considered as an optical MEMS) is well-suited for eXterm Adaptive Optics architecture as a "tweeter" correction (i.e. high-order correction with small dynamic). Indeed, low-order wavefront correction (up to $Z_{36}$ ) with $f<1 \mathrm{KHz}$ with a classical piezoelectric DM as a "boomer" deformable mirror is needed to desaturate the wavefront correction. This configruation can be applied for the E-ELT AO system (Hubin et al. 2006).

Acknowledgements. This work received the support of the University of Liège. The author is grateful to T. Nakos (ESO) for the manuscript corrections. The author also thank Antoine Labeyrie, for helpful discussions concerning the cavity optimization. The author wishes to thank the anonymous referee for useful comments and corrections.

\section{Appendix A: Waffle effect: the Fresnel propagation}

If the Fresnel diffraction pattern of a simple slit is well-defined, we obtain

$$
\begin{aligned}
& U_{i, j}\left(\xi, x^{\prime}, y^{\prime}\right)=\frac{\mathrm{e}^{\mathrm{i} 2 \pi z / \lambda}}{2 i}\left[\left(C\left(\xi_{x 2}\right)+i S\left(\xi_{x 2}\right)-C\left(\xi_{x 1}\right)-i S\left(\xi_{x 1}\right)\right)\right] \\
& \quad \times\left[\left(C\left(\xi_{y 2}\right)+i S\left(\xi_{y 2}\right)-C\left(\xi_{y 1}\right)-i S\left(\xi_{y 1}\right)\right],\right.
\end{aligned}
$$

where $z$ is the propagation distance between the microlens array and the membrane, $\lambda$ is the laser wavelength, $x^{\prime}, y^{\prime}$ is the cartesian coordinate of the diffracted field. $C(\xi) / S(\xi)$ are the classical Fresnel integrals defined by

$$
C(\xi)=\int_{0}^{\xi} \cos \left(\pi t^{2} / 2\right) \mathrm{d} t \quad \text { and } \quad S(\xi)=\int_{0}^{\xi} \sin \left(\pi t^{2} / 2\right) \mathrm{d} t
$$

where $\xi$ are the diffraction parameters for each laser diode, given by

$$
\begin{aligned}
& \xi_{x 2}^{V}=\sqrt{8 N_{x}^{V}}\left(\frac{1}{2}-\frac{x^{\prime}+x_{i, j}+\Lambda_{x} / 2}{\left(\Lambda_{x}-F_{x}\right)}\right) \\
& \xi_{x 1}^{V}=-\sqrt{8 N_{x}^{V}}\left(\frac{1}{2}+\frac{x^{\prime}+x_{i, j}+\Lambda_{x} / 2}{\left(\Lambda_{x}-F_{x}\right)}\right) \\
& \xi_{y 2}^{V}=\sqrt{8 N_{y}^{V}}\left(\frac{1}{2}-\frac{y^{\prime}+y_{i, j}+\Lambda_{y} / 2}{\Lambda_{y}}\right) \\
& \xi_{y 1}^{V}=-\sqrt{8 N_{y}^{V}}\left(\frac{1}{2}+\frac{y^{\prime}+y_{i, j}+\Lambda_{y} / 2}{\Lambda_{y}}\right) \\
& \xi_{x 2}^{H}=\sqrt{8 N_{x}^{H}}\left(\frac{1}{2}-\frac{x^{\prime}+x_{i, j}+\Lambda_{x} / 2}{\Lambda_{x}}\right) \\
& \xi_{x 1}^{H}=-\sqrt{8 N_{x}^{H}}\left(\frac{1}{2}+\frac{x^{\prime}+x_{i, j}+\Lambda_{x} / 2}{\Lambda_{x}}\right) \\
& \xi_{y 2}^{H}=\sqrt{8 N_{y}^{H}}\left(\frac{1}{2}-\frac{y^{\prime}+y_{i, j}+\Lambda_{y} / 2}{\left(\Lambda_{y}-F_{y}\right)}\right) \\
& \xi_{y 1}^{H}=-\sqrt{8 N_{y}^{H}}\left(\frac{1}{2}+\frac{y^{\prime}+y_{i, j}+\Lambda_{y} / 2}{\left(\Lambda_{y}-F_{y}\right)}\right)
\end{aligned}
$$


where $N_{x}^{V}, N_{y}^{V}, N_{x}^{H}, N_{y}^{H}$ are the four Fresnel numbers

$N_{x}^{V}=\frac{\left(\Lambda_{x}-F_{x}\right)^{2}}{4 \lambda z} \quad N_{y}^{V}=\frac{\Lambda_{y}^{2}}{4 \lambda z}$

$N_{x}^{H}=\frac{\Lambda_{x}^{2}}{4 \lambda z} \quad N_{y}^{H}=\frac{\left(\Lambda_{y}-F_{y}\right)^{2}}{4 \lambda z}$

The total illuminated Fresnel field has an amplitude of $U_{\mathrm{f}}=$ $U_{\mathrm{g}}+U_{\mathrm{Fr}}$, where $U_{\mathrm{g}}$ is the direct field and $U_{\mathrm{Fr}}$ the diffracted field. Each term must be calculated as follows:

$U_{\mathrm{g}}=\sum_{i=1}^{n_{x}} \sum_{j=1}^{n_{y}} U_{i, j}^{\mathrm{L}}$

$e_{i, j}^{V}=\frac{\int_{y=-\Lambda_{y} / 2}^{y=\Lambda_{y} / 2}\left|U_{\mathrm{g}}\left(x_{i, j}+\frac{\Lambda_{x}}{2}, y_{i, j}+y\right)\right|^{2} \mathrm{~d} y}{\int_{-\infty}^{+\infty} \int_{-\infty}^{+\infty}\left|U_{\mathrm{g}}\right|^{2} \mathrm{~d} x \mathrm{~d} y}$

$e_{i, j}^{H}=\frac{\int_{x=-\Lambda_{x} / 2}^{x=\Lambda_{x} / 2}\left|U_{\mathrm{g}}\left(x_{i, j}+x, y_{i, j}+\frac{\Lambda_{y}}{2}\right)\right|^{2} \mathrm{~d} x}{\int_{-\infty}^{+\infty} \int_{-\infty}^{+\infty}\left|U_{\mathrm{g}}\right|^{2} \mathrm{~d} x \mathrm{~d} y}$

and

$$
\begin{aligned}
U_{\mathrm{Fr}}= & \sum_{i=1}^{n_{x}-1} \sum_{j=1}^{n_{y}-1}\left[U_{i, j}\left(\xi^{V}\right) e_{i, j}^{V}+U_{i, j}\left(\xi^{H}\right) e_{i, j}^{H}\right] \\
& +\sum_{j=1}^{n_{y}}\left[U_{0, j}\left(\xi^{V}\right) e_{0, j}^{V}+U_{n_{x}, j}\left(\xi^{V}\right) e_{n_{x}, j}^{V}\right] \\
& +\sum_{i=1}^{n_{x}}\left[U_{i, 0}\left(\xi^{H}\right) e_{i, 0}^{H}+U_{i, n_{y}}\left(\xi^{H}\right) e_{i, n_{y}}^{H}\right],
\end{aligned}
$$

where $e_{i, j}^{V}$ and $e_{i, j}^{H}$ are the light intensity values (energy) inside the vertical and horizontal slits, respectively.

\section{Appendix B: Linear model for the static solutions}

In the first approximation (linear model), the deformable mirror can be well modeled by the plate equation of Timoshenko \& Woinowsky-Krieger (1959):

$$
D \nabla^{4} w-\frac{P_{t}}{a b} \nabla^{2} w=P_{z}(x, y) \quad D=\frac{E h^{3}}{12\left(1-v^{2}\right)},
$$

where $\nabla^{4}$ and $\nabla^{2}$ are the biharmonic and the nabla operators on the deformation $w(x, y)$, respectively. $P_{z}(x, y)$ is the radiation pressure, and $P_{t}$ is the membrane tension. For the numerical simulation, we chose a value of $P_{t}=0.25 \mathrm{~N} / \mathrm{m}$ (low mechanical tension). The flexural rigidity $D$ depends on the Young's modulus $E$, the Poisson's ratio $v$ and the membrane thickness $h$. We saw in Sect. 5.3 how the full membrane structure ( $\mathrm{Al} /$ diamond/Al or $\mathrm{Al} /$ diamond/Au) can be taken into account.

Using Navier's method, the plate Eq. (B.1) can be transformed into a set of two linear partial differential equations:

$\frac{\partial^{2} M}{\partial x^{2}}+\frac{\partial^{2} M}{\partial y^{2}}=-P_{z}(x, y) \quad$ and $\quad D\left(\frac{\partial^{2} w}{\partial x^{2}}+\frac{\partial^{2} w}{\partial y^{2}}\right)=-M$

where $M$ is the moment sum.

\section{B.1. Boundary conditions and periodic solutions}

Owing to the actuator influence function given by the microlens array focal ratio, the wavefront correction shape $w(x, y)$ is generally greater than $n_{x} \cdot \Lambda_{x} \times n_{y} \cdot \Lambda_{y}$ in surface (see Fig. 3 ). The general boundary condition reads

$\begin{array}{llll}M(x=0)=0 & M(x=a)=0 & w(x=0)=0 & w(x=a)=0 \\ M(y=0)=0 & M(y=a)=0 & w(y=0)=0 & w(y=a)=0,\end{array}$

where $a$ and $b$ are the geometrical extensions in the $\mathrm{x}$ and $\mathrm{y}$ axis of the single large actuator used in this mechanical simulation, respectively. Because of the intrinsic influence actuator function, the DM active surface extension $a>n_{x} \Lambda_{x}$ and $b>n_{y} \Lambda_{y}$. To include in the $w$ deformation function the $n_{x} \times n_{y}$ actuators, we chose a sinusoidal periodic orthogonal set of functions that satisfies these boundary conditions (Fourier decomposition):

$w(x, y)=\sum_{m=1}^{n_{x}} \sum_{n=1}^{n_{y}} W_{m, n} S_{m}(x) S_{n}(y)$
$S_{m}(x)=\sin \left(\frac{m \pi x}{a}\right) \quad S_{n}(y)=\sin \left(\frac{n \pi y}{b}\right)$.

The orthogonal set of functions possess the following properties:

$$
\begin{aligned}
& \int_{0}^{a} S_{m}(x) S_{m^{\prime}}(x) \mathrm{d} x=0 \quad\left(m^{\prime} \neq m\right)=a / 2 \quad\left(m^{\prime}=m\right) \\
& \int_{0}^{b} S_{n}(y) S_{n^{\prime}}(y) \mathrm{d} y=0 \quad\left(n^{\prime} \neq n\right) \quad=b / 2 \quad\left(n^{\prime}=n\right) \\
& W_{m, n}=\frac{4}{a b} \int_{0}^{a} \int_{0}^{b} w(x, y) S_{m}(x) S_{n}(y) \mathrm{d} x \mathrm{~d} y
\end{aligned}
$$

\section{Appendix C: Multi-material mechanical transformations}

The following equations show the different transformations applied on the $D, v, E$ coefficients for two materials:

$$
\begin{aligned}
& D_{\mathrm{e}}=\frac{E_{1} h_{1}^{3}}{12\left(1-v_{1}^{2}\right)} K_{\beta} \quad v_{\mathrm{e}}=v_{1} \frac{K_{\gamma}}{K_{\beta}} \quad E_{\mathrm{e}}=\frac{D_{\mathrm{e}}\left(1-v_{\mathrm{e}}^{2}\right)}{\left(h_{1}+h_{2}\right)^{3}} \\
& K_{\beta}=1+\frac{E_{2} h_{2}^{2}\left(1-v_{1}^{2}\right)}{E_{1} h_{1}^{2}\left(1-v_{2}^{2}\right)}+\frac{3\left(1-v_{1}^{2}\right)\left(1+\frac{h_{2}}{h_{1}}\right)^{2}\left(1+\frac{E_{1} h_{1}}{E_{2} h_{2}}\right)}{\left(1+\frac{E_{1} h_{1}}{E_{2} h_{2}}\right)^{2}-\left(v_{1}+v_{2} \frac{E_{1} h_{1}}{E_{2} h_{2}}\right)^{2}} \\
& K_{\gamma}=1+\frac{v_{2} E_{2} h_{2}^{3}\left(1-v_{1}^{2}\right)}{v_{1} E_{1} h_{1}^{3}\left(1-v_{2}^{2}\right)}+\frac{3\left(1-v_{1}^{2}\right)\left(1+\frac{h_{2}}{h_{1}}\right)^{2}\left(1+\frac{v_{2} E_{1} h_{1}}{v_{1} E_{2} h_{2}}\right)}{\left(1+\frac{E_{1} h_{1}}{E_{2} h_{2}}\right)^{2}-\left(v_{1}+v_{2} \frac{E_{1} h_{1}}{E_{2} h_{2}}\right)^{2}}
\end{aligned}
$$

The $K_{\gamma}$ coefficient is equivalent to $K_{3 p}$ in Myers et al. (2006) but with an error correction.

\section{Appendix D: Nonlinear model for the static solutions}

Our surface deflection amplitude can be more than the membrane thickness. In this case, the vertical deflections are accompanied by stretching of the mirror surface. The stretching is a nonlinear process that causes the mirror layer to increase 
its load-carrying capabilities. Now, our model possesses a new stress function $G\left(\phi_{s}, \sigma\right)$ defined as

$$
\begin{aligned}
\nabla^{4} \phi_{s}=- & \frac{E_{\mathrm{e}}}{2} G(w, 0) \\
G\left(\phi_{s}, \sigma\right)= & \frac{\partial^{2} w}{\partial x^{2}}\left(\frac{\partial^{2} \phi_{s}}{\partial y^{2}}+\sigma_{x}\right)+\frac{\partial^{2} w}{\partial y^{2}}\left(\frac{\partial^{2} \phi_{s}}{\partial x^{2}}+\sigma_{y}\right) \\
& -2 \frac{\partial^{2} w}{\partial x \partial y}\left(\frac{\partial^{2} \phi_{s}}{\partial x \partial y}+\sigma_{x y}\right) \\
\frac{G(w, 0)}{2}= & {\left[\left(\frac{\partial^{2} w}{\partial x^{2}} \frac{\partial^{2} w}{\partial y^{2}}-\frac{\partial^{2} w}{\partial x \partial y} \frac{\partial^{2} w}{\partial x \partial y}\right)\right] }
\end{aligned}
$$

where $\phi_{s}$ is an Airy-type stress function and $\sigma=\sigma_{x}=\sigma_{y}=\sigma_{x y}$ is a stress constant. We used the value equal to $20 \mathrm{MPa}$ for this application (see Table 3). Therefore, this term can be moved to the left hand side of Eq. (29). We notice here that we used the stiffness and the Young's modulus for a composite membrane.

\section{D.1. $\phi_{s}(x, y)$ Airy stress function}

We express the $\phi_{s}$ stress function in the same basis set as the $w(x, y)$ function (Fourier decomposition).

$\phi_{s}(x, y)=\sum_{m=1}^{n_{x}} \sum_{n=1}^{n_{y}} \phi_{s, m, n} C_{m}(x) C_{n}(y)$
$C_{m}(x)=\cos \left(\frac{m \pi x}{a}\right) \quad C_{n}(y)=\cos \left(\frac{n \pi y}{b}\right)$.

The Airy-type stress function solves in the same manner as the $w(x, y)$ deflection function with the Galerkin method (see Azucena et al. 2008; Timoshenko \& Woinowsky-Krieger 1959, p. 425). We obtain the following equations:

$$
\begin{gathered}
\int_{0}^{a} \int_{0}^{b}\left(\nabla^{4} \phi_{s}+\frac{E_{\mathrm{e}}}{2} G(w, 0)\right) C_{m}(x) C_{n}(y) \mathrm{d} x \mathrm{~d} y=0 \\
\nabla^{4} \phi_{s}=\sum_{m=1}^{n_{x}} \sum_{n=1}^{n_{y}}\left(\left(\frac{m \pi}{a}\right)^{2}+\left(\frac{n \pi}{b}\right)^{2}\right)^{2} \phi_{s, m, n} C_{m}(x) C_{n}(y) .
\end{gathered}
$$

The derivative of $w(x, y)$ deflection function can be expressed in the following form:

$$
\begin{aligned}
& \frac{\partial^{2} w}{\partial x^{2}}=\sum_{r=1}^{n_{x}} \sum_{s=1}^{n_{y}} \frac{-r^{2} \pi^{2}}{a^{2}} W_{r, s} S_{r}(x) S_{s}(y) \\
& \frac{\partial^{2} w}{\partial y^{2}}=\sum_{p=1}^{n_{x}} \sum_{q=1}^{n_{y}} \frac{-q^{2} \pi^{2}}{b^{2}} W_{p, q} S_{p}(x) S_{q}(y) \\
& \frac{\partial^{2} w}{\partial x \partial y}=\sum_{r=1}^{n_{x}} \sum_{s=1}^{n_{y}} \sum_{p=1}^{n_{x}} \sum_{q=1}^{n_{y}} \frac{r s p q \pi^{4}}{a^{2} b^{2}} \\
& W_{r, s} W_{p, q} S_{r}(x) S_{s}(y) S_{p}(x) S_{q}(y) .
\end{aligned}
$$

The orthogonal set of functions $C_{m} / C_{n}$ possess the same properties asn $S_{m} / S_{n}$,

$$
\begin{aligned}
& \int_{0}^{a} C_{m}(x) C_{m^{\prime}}(x) d x=0 \quad\left(m^{\prime} \neq m\right)=a / 2 \quad\left(m^{\prime}=m\right)(\mathrm{D} .11) \\
& \int_{0}^{b} C_{n}(y) C_{n^{\prime}}(y) d y=0 \quad\left(n^{\prime} \neq n\right) \quad=b / 2 \quad\left(n^{\prime}=n\right) .
\end{aligned}
$$

Table D.1. $B_{r s p q}$ and $C_{r s p q}$ coefficients.

\begin{tabular}{lcc}
\hline \hline$B_{r s p q} / C_{r s p q}$ & $r+p=m$ & $r-p=m$ \\
\hline$s+q=n$ & $-(r q-s p)^{2} /-(r q-s p)^{2}$ & $(r q+s p)^{2} /(r q+s p)^{2}$ \\
$s-q=n$ & $(r q+s p)^{2} /-(r q+s p)^{2}$ & $-(r q-s p)^{2} /(r q-s p)^{2}$ \\
\hline
\end{tabular}

Now, because of the compling coefficients $W_{r, s} W_{p, q}$ this is necessary to calculate during the final integration:

$$
\int_{0}^{a} C_{m}(x) S_{r}(x) S_{p}(x) \mathrm{d} x=0 \quad(r \pm p \neq m)
$$

$$
\int_{0}^{a} C_{m}(x) S_{r}(x) S_{p}(x) \mathrm{d} x=\mp a / 4 \quad(r \pm p=m)
$$

$\int_{0}^{a} C_{m}(x) C_{r}(x) C_{p}(x) \mathrm{d} x=0 \quad(r \pm p \neq m)$

$$
\int_{0}^{a} C_{m}(x) C_{r}(x) C_{p}(x) \mathrm{d} x=a / 4 \quad(r \pm p=m) .
$$

We obtain the same kinds of results $( \pm b / 4)$ with the integation on the $y$ axis on the $s$ and $q$ variables as Eq. (D.13). After simplifications, the Airy stress function can be calculated by correlating the input $w(x, y)$ deformation fonction (see Table D.1), and we obtain:

$$
\phi_{s, m, n}=\frac{E_{\mathrm{e}}}{4\left(m^{2} b / a+n^{2} a / b\right)^{2}} \sum B_{r s p q} W_{r, s} W_{p, q}
$$

The Airy stress function presents a strong spatial frequency dependence: the most part of the Stress function is in low frequency (see Fig. 4). This result presents some limitations on the analytical evaluation of $\phi_{s, m, n}$. Indeed, the real area of the membrane is $\pi r_{m}^{2}$ not only $a b$ that used in the previous calculation for the active area of the DM. This physical characteristic changes the real value of the Airy stress function by frequency filtering. The $\phi_{s, m, n}$ must be evaluated on the entire circular membrane and then estimated on the active area of the DM. The solution presented here seems to be pessimistic but a full analytical development is beyond the scope of this paper. A global finite elements model (FEM) of the DM seems to be more useful to know the dynamical response with respect to the entrance VCSEL laser power.

\section{D.2. $G\left(\phi_{s}, \sigma\right)$ equation}

The $G\left(\phi_{s}, \sigma\right)$ must be treated in the same manner,

$G\left(\phi_{s}, \sigma\right)=G(0, \sigma)+G\left(\phi_{s}, 0\right)$.

Equation $G(0, \sigma)$ becomes

$G(0, \sigma)=\frac{-\sigma \pi^{2}}{a b}\left(m^{2} b / a+n^{2} a / b\right) W_{m, n}$.

Following the mathematical development in the system of Eq. (D.17), the function $G\left(\phi_{s}, 0\right)$ gives

$G\left(\phi_{s}, 0\right)=\frac{\pi^{4}}{4 a^{2} b^{2}} \sum C_{r s p q} \phi_{s, r, s} W_{p, q}$. 


\section{Appendix E: Analytical evaluation of $I_{z, m, n}$}

For a purely Gaussian laser field (without the Fresnel propagation), the integal $I_{z, m, n}$ can be evaluated analytically as a convolution system $\left(I_{i, j}^{\mathrm{L}} \otimes\right.$ Gaussian $)$ :

$$
\begin{aligned}
& I_{z, m, n} \approx \frac{4\left(\left|r_{1}\right|^{2}-A_{1}\right)}{c a^{2} b^{2}} \sum_{m=1}^{n_{x}} \sum_{n=1}^{n_{y}} I_{m, n}^{\mathrm{L}} E_{\mathrm{g} x y}(a, b, m, n) \\
& I_{m, n}^{\mathrm{L}}=\int_{0}^{a} \int_{0}^{b} I_{i, j}^{\mathrm{L}} \delta\left(x-x_{i, j}\right) \delta\left(y-y_{i, j}\right) S_{m}(x) S_{n}(y) \mathrm{d} x \mathrm{~d} y \\
& E_{\mathrm{g} x y}(a, b, m, n)=\int_{0}^{a} \int_{0}^{b} \mathrm{e}^{\frac{-\left((x-a / 2)^{2}+(y-b / 2)^{2}\right)}{2\left(\sigma_{\mathrm{g} x}^{2}+\sigma_{\mathrm{g} y}^{2}\right)}} S_{m}(x) S_{n}(y) \mathrm{d} x \mathrm{~d} y \\
& E_{\mathrm{g} x y}(a, b, m, n)=-\frac{\pi}{8} \mathrm{e}^{-\frac{\pi^{2}\left(\sigma_{\mathrm{g} x}^{2}+\sigma_{\mathrm{g} x}^{2}\right)\left(b^{2} m^{2}+a^{2} n^{2}\right)+i b^{2} \pi(m+n)}{2 a^{2} b^{2}}} \\
& \times\left(\sigma_{\mathrm{g} x}^{2}+\sigma_{\mathrm{g} x}^{2}\right)\left(\mathrm{e}^{\mathrm{i} m \pi}-1\right)\left(\mathrm{e}^{\mathrm{i} n \pi}-1\right) \\
& \times\left(\operatorname{Erf}\left(\frac{a^{2}+2 \mathrm{i} m \pi\left(\sigma_{\mathrm{g} x}^{2}+\sigma_{\mathrm{g} y}^{2}\right)}{2 a \sqrt{2\left(\sigma_{\mathrm{g} x}^{2}+\sigma_{\mathrm{g} x}^{2}\right)}}\right)+\operatorname{Erf}\left(\frac{a^{2}-2 \mathrm{i} m \pi\left(\sigma_{\mathrm{g} x}^{2}+\sigma_{\mathrm{g} y}^{2}\right)}{2 a \sqrt{2\left(\sigma_{\mathrm{g} x}^{2}+\sigma_{\mathrm{g} x}^{2}\right)}}\right)\right) \\
& \times\left(\operatorname{Erf}\left(\frac{b^{2}+2 \mathrm{i} n \pi\left(\sigma_{\mathrm{g} x}^{2}+\sigma_{\mathrm{g} y}^{2}\right)}{2 b \sqrt{2\left(\sigma_{\mathrm{g} x}^{2}+\sigma_{\mathrm{g} x}^{2}\right)}}\right)+\operatorname{Erf}\left(\frac{b^{2}-2 \mathrm{i} n \pi\left(\sigma_{\mathrm{g} x}^{2}+\sigma_{\mathrm{g} y}^{2}\right)}{2 b \sqrt{2\left(\sigma_{\mathrm{g} x}^{2}+\sigma_{\mathrm{g} x}^{2}\right)}}\right)\right),
\end{aligned}
$$

where $\operatorname{Erf}$ is the error function (the integral of the Gaussian distribution). Numerically, we verify that $E_{\mathrm{g} x y} \in \mathfrak{R}$.

To retrieve the exact value of the illuminated laser field $I_{m, n}^{\mathrm{L}}$, we must deconvolve in the Fourier plane the $I_{z, m, n}$ illumination obtained by our model using the $E_{\mathrm{g} x y}$ function. Indeed, $I_{m, n}^{\mathrm{L}} \neq I_{z, m, n} / E_{\mathrm{g} x y}$ and this operation is very similar to the myopic deconvolution given by Conan et al. (1998).

\section{References}

Azucena, O., Fernandez, B., \& Kubby, J. 2008, in MEMS Adaptive Optics II, eds. S. S. Olivier, T. G. Bifano, \& J. A. Kubby, Proc. SPIE, 6888, 68880Q

Bardinal, V., Camps, T., Reig, B., et al. 2011, Adv. Opt. Tech., 2011, ID 609643 Barron, L. 2005, High-Reflectance, Sputter-Deposited Aluminum Alloy Thin Films for Micro-Electro-Mechanical Systems, Master Thesis
Bass, M., DeCusatis, C., Enoch, J., et al. 2009, Handbook of Optics, Vol. IV, Optical Properties of Materials, Nonlinear Optics, Quantum Optics (McGraw-Hill), 1152

Conan, J.-M., Mugnier, L. M., Fusco, T., Michau, V., \& Rousset, G. 1998, Appl. Opt., 37, 4614

Conk, R. 2002, Fabrication techniques for micro-optical device arrays, Master Thesis

Daughton, D. R., MacDonald, J., \& Mulders, N. 2003, J. Non-Cryst. Sol., 319, 297

Dubey, Y. 2005, An approximate solution to buckling of plates by the galerkin method, Master Thesis

Espinosa, H., Peng, B., Prorok, B., et al. 2003, J. Appl. Phys., 94, 6076

Galerkin, B. 1915, Vestnik inzhenerov i tekhnikov, 19, 897

Hess, P. 2012, J. Appl. Phys., 111, 051101

Hubin, N., Ellerbroek, B. L., Arsenault, R., et al. 2006, in The Scientific Requirements for Extremely Large Telescopes, eds. P. Whitelock, M. Dennefeld, \& B. Leibundgut, IAU Symp., 232, 60

Kasten, A., Giannopoulos, A., Long, C., C., C., \& Choquette, K. 2007, in Proc. SPIE, 6484, 64840C

Kerrour, F., \& Hobar, F. 2006, Semiconductor Physics, Quant. Electron. \& Optoelectron., 9, 52

Koizumi, S., Nebel, C., \& Nesládek, M. 2008, Physics and Applications of CVD Diamond (Wiley-VCH), 374

Labeyrie, A., Coroller, H. L., Residori, S., et al. 2010, in Pathways Towards Habitable Planets, eds. V. Coudé Du Foresto, D. M. Gelino, \& I. Ribas, ASP Conf. Ser., 430, 239

Lee, J.-H., Lee, Y.-C., \& Kang, E.-C. 2006, J. Opt. Soc. Korea, 10, 57

Magette, A. 2010, Ph.D. Thesis: The International Liquid Mirror Telescope: optical testing and alignment using a Nijboer-Zernike aberration retrieval approch, IAGL, University of Liège, 1

Mansuripur, M. 2004, Opt. Expr., 12, 5375

Mearini, G. 1992, Ph.D. Thesis, Case Western Reserve University

Mehta, P. 2009, Vibrations of thin plate with piezoelectric actuator: theory and experiments, Master Thesis

Morse, K. A., McHugh, S. L., \& Fixler, J. 2008, Appl. Opt., 47, 5325

Myers, O., Anjanappa, M., \& Freidhoff, C. 2006, COMSOL Users Conference, Boston, MA, October, 1

Pandey, A. K., Venkatesh, K., \& Pratap, R. 2009, Sādhanāa, 34, 651

Salvadori, M. C., Brown, I. G., Vaz, A. R., Melo, L. L., \& Cattani, M. 2003, Phys. Rev. B, 67, 153404

Seurin, J., Ghosh, C., Khalfin, V., et al. 2008, in Vertical-Cavity Surface-Emitting Lasers XII, eds. C. Lei, \& J. K. Guenter, Proc. SPIE, 6908, 69080

Sheplak, M., \& Seiner, J. 1999, in 37th AIAA Aerospace Sciences Meeting, Reno

Sinha, S., Mansell, J. D., \& Byer, R. L. 2002, in Proc. SPIE, 4493, 55

Thornell, G., Ericson, F., Hedlund, C., et al. 1999, in Am. Phys. Soc., 981

Timoshenko, S. P., \& Woinowsky-Krieger, S. 1959, Theory of Plates and Shells 2nd edition (McGraw-Hill), 594

Trauger, J. T. 2009, in BAAS, 41, AAS Meeting Abstracts, 213, 234.04

Zimmermann, A., Gross, J., \& Fricke, J. 1995, J. Non-Cryst. Sol., 186, 238, proceedings of the Fourth International Symposium on AEROGELS 